\title{
High cycle thermal fatigue of austenitic stainless steel
}

\author{
Yanjun Wang ${ }^{1,2}$, Ali Charbal ${ }^{1,2,+}$, François Hild ${ }^{2}$ and Ludovic Vincent ${ }^{1, *}$ \\ ${ }^{1}$ DEN-Service de Recherches Métallurgiques Appliquées, CEA, Université Paris-Saclay, 91191, Gif sur Yvette, France \\ ${ }^{2}$ LMT, ENS Paris-Saclay, CNRS, Université Paris-Saclay, 61 av. du Président Wilson, 94235 Cachan cedex, France \\ now at Lehigh University, Packard Lab, 19 Memorial Drive West, Bethlehem, PA 18015, USA
}

\begin{abstract}
A new experimental setup called FLASH (THErmal Fatigue by LASer or Helium pulses) has been developed to perform thermal fatigue tests on materials used in structures of Sodium-cooled Fast Reactors (SFRs). A set of thermal loadings ranging from $150^{\circ} \mathrm{C}$ to $200^{\circ} \mathrm{C}$ prescribed by a high energy laser has been applied to $\mathrm{A} 316 \mathrm{~L}(\mathrm{~N})$ stainless steel samples. Thermal fatigue tests are performed until a macrocrack is formed. The 3D displacement fields of the surface impacted by the laser beam are measured by a hybrid multiview system. A thermomechanical model is used to compute displacement fields that are compared with correlation measurements. These results are compared in terms of strain levels to the fatigue curve determined from standardized isothermal uniaxial mechanical fatigue tests.
\end{abstract}

\section{Introduction}

In a sodium cooled fast nuclear reactor, the primary pump circulates cold sodium $\left(400^{\circ} \mathrm{C}\right)$ between fuel assemblies to remove the heat resulting from fission reactions. Several heat exchangers then transfer this energy from hot sodium $\left(550^{\circ} \mathrm{C}\right)$ to steam generators in order to drive the turbines that eventually produce electricity [1]. There are different conditions where repeated thermal shocks may occur in the component walls of the reactor made of AISI $316 \mathrm{~L}(\mathrm{~N})$ austenitic stainless steel. When these loadings are repeated over a large number of cycles, initiation and propagation of a crack network may be induced at the surface of the components, which is referred to as thermal striping [2]. Design codes in the nuclear industry deal with thermal fatigue considering an equivalent strength (i.e. mechanical strain amplitude) as in standardized isothermal strain controlled push-pull mechanical fatigue tests. From previous thermal fatigue results obtained at CEA [3, 4], it appeared that this approach could be nonconservative, namely, the number of cycles to crack initiation in thermal fatigue experiments could be lower than in push-pull isothermal fatigue tests for the same levels of equivalent strain amplitude.

The present work focuses on the determination of crack initiation under thermal fatigue using the FLASH facility, which allows high cycle (about several $10^{6}$ ) thermal fatigue tests to be performed with excellent stability of the thermal loading [5]. A pulsed laser is used to prescribe cyclic heat fluxes onto the surface of austenitic stainless steel samples. Thanks to the separation of the wavelengths of the laser $(1.064 \mu \mathrm{m})$ on the one hand and of an infrared camera [3.97-4.01 $\mu \mathrm{m}]$ and two large-band pyrometers $[1.55-2.2 \mu \mathrm{m}]$ on the other hand, measurements of the temperature fields are performed on the surface area of the specimens impacted by the laser where temperature variations are the largest and where crack initiation eventually occurs. This setup greatly improves the signal/noise ratio generally obtained in thermal fatigue experiments and then produces better estimations of the boundary conditions of the thermomechanical simulations required to interpret such tests. With the predictions of finite element analyses, it is deduced that the largest strain variations during cyclic loading are in the out-of-plane direction resulting in several micrometer out-of-plane displacements [6]. The 3D displacement fields are then measured by a hybrid multiview system composed of two visible light cameras and the infrared camera that also gives access to the Lagrangian temperature fields [7, 8]. Last, the equivalent Tresca strain variation obtained from finite element analyses is extracted in the region of crack initiation. This estimation is compared with results obtained from standardized isothermal uniaxial mechanical fatigue tests.

\section{Experimental setup}

FLASH is designed with the purpose of generating localized cyclic thermal shocks by pulsed laser or helium jets that are able to reproduce crack initiation in laboratory conditions (Fig. 1). The specimens are plates made of $316 \mathrm{~L}(\mathrm{~N})$ austenitic stainless steel $(270 \times 40 \times$ $7 \mathrm{~mm}^{3}$ ), which are continuously heated by an electrical current (Joule effect) until their center reaches $400^{\circ} \mathrm{C}$, a temperature (controlled by a K-type thermocouple) representative of the cold primary sodium temperature. Static 4-point bend loading can be added so that the thermally shocked region will undergo a non-zero mean uniaxial stress.

* Corresponding author: ludovic.vincent $@$ cea.fr 


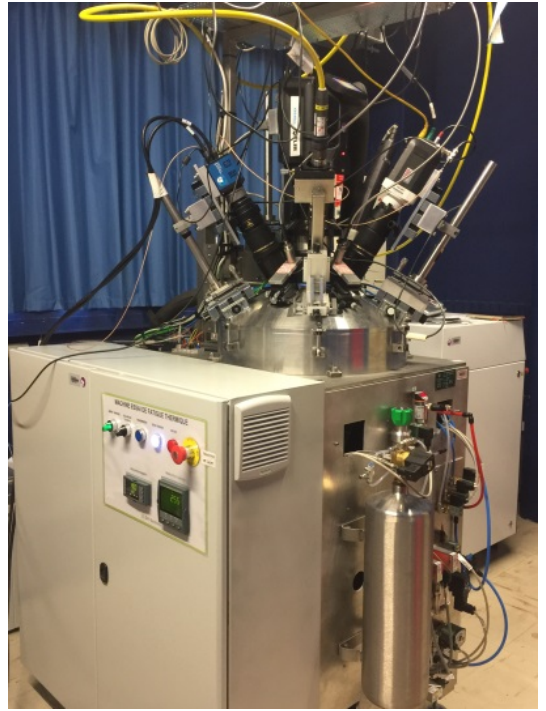

Fig. 1. Overview of FLASH facility

The thermal loadings are performed by a pulsed laser (TruPulse 156, Trumpf, $\lambda=1064 \mathrm{~nm}$, see Fig. 2-(1)) on the center of specimen at a frequency of $1 \mathrm{~Hz}$, the pulse duration is $50 \mathrm{~ms}$ and the incident pulsed power is adapted to obtain the desired temperature variation on the surface of the specimen. A focusing optics allows a top-hat power density distribution to be obtained over a $5-\mathrm{mm}$ in diameter disk. Due to the relatively low absorptivity of the polished and pre-oxidized surface, the laser beam is tilted to be reflected toward a power detector that gives access to the mean power reflected by the specimen surface.

An infrared camera (x6540sc FLIR, definition: $640 \times 512$ pixels, $\lambda \in[3,5 \mu \mathrm{m}]$ reduced to $\lambda \in$ $[3.97,4.01 \mu \mathrm{m}]$ with an internal filter for high temperature measurements, Fig. 2.-(2)) is used to measure the 2D temperature field and the 3D displacement fields with a high magnification lens allowing for a pixel resolution of $15 \mu \mathrm{m}$. Two fast pyrometers (KGA740-LO, $\lambda \in[3,5 \mu m]$, Fig. 2.-(3)) are used to measure and monitor within the impacted zone the temperature variation on a $2.5 \mathrm{~mm}$ central area. The first visible light camera (MIRO M320S, Vision Research, definition: $1920 \times 1080$ pixels, Fig. 2.-(4)) with a pixel resolution of $10 \mu \mathrm{m}$ corresponds to the second device of the multiview system. Another visible light camera was recently introduced as the third device (pco.edge, definition: $2560 \times 2160$ pixels, Fig. 2.-(5)) with a higher pixel resolution of $6.5 \mu \mathrm{m}$. All three cameras are synchronized on the same stroboscopic acquisition signal to compensate for the relatively lower acquisition frequency of the pco.edge camera, and at the same time to facilitate the comparison between simulated and experimental thermomechanical fields. The specimen is put inside an airtight chamber filled with helium atmosphere to eliminate possible excessive oxidation that would alter the surface emissivity, namely, the speckles to enable for DIC analyses, the heat exchange between the specimen and the laser beam (absorptivity) or helium atmosphere (convection).

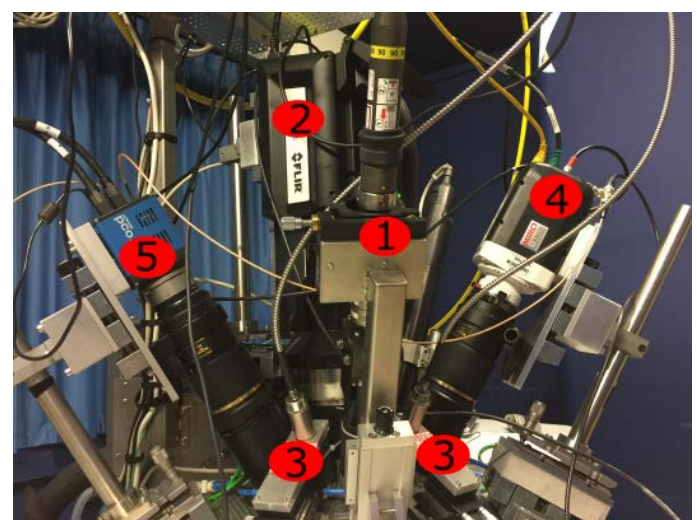

Fig. 2. Experimental configuration with principal components (see main text for a detailed description of the components labelled in the picture)

\section{Thermomechanical analyses}

Three-dimensional finite element simulations are carried out with the assumption that the thermal and mechanical loadings are uncoupled. Such numerical simulations are necessary to interpret the results of the thermal fatigue test through equivalent strain variations (scalar) built from complete strain tensors. These simulations also give access to the $3 \mathrm{D}$ displacement field on the specimen surface impacted by the laser beam, which can be compared with the multiview correlation measurements. First, a thermal analysis is performed with the purpose of reproducing the temperature fields measured during cyclic loadings. Once the cyclic history of temperature at the shock center is stabilized, then the temperature field of the last cycle is used as an input of the thermomechanical calculation. Due to geometrical symmetry, only one quarter of the FLASH specimen (which corresponds to a simulation volume of $105 \times 20 \times$ $7 \mathrm{~mm}^{3}$ ) is modeled to minimize the computation time and numerical storage.

\subsection{Heat transfer analyses}

The purpose of this section is to present the finite element model of heat transfer to evaluate the temperature fields. The spatiotemporal temperature field is determined considering conductive and convective hear transfer processes

$$
\rho c_{p} \frac{\partial}{\partial t} T(\mathbf{x}, t)=k \Delta T(\mathbf{x}, t)+s(\mathbf{x})
$$

where $T$ is the temperature distribution in the specimen, $\Delta$ the Laplacian, $\rho$ the mass density $\left(\mathrm{kg} \cdot \mathrm{m}^{-3}\right), c_{p}$ the specific heat capacity $\left(J \cdot \mathrm{kg}^{-1} \cdot \mathrm{K}^{-1}\right), k$ the thermal conductivity $\left(W \cdot m^{-1} \cdot K^{-1}\right)$, and $s$ the volumetric heat source accounting for the heat flux induced by Joule effect. Other thermophysical parameters of the material are chosen from data reported in the French design code RCC-MRx [9]. The natural convection flux across the boundary layer on the surface of the specimen reads

$$
q=-h\left(T-T_{H e}\right)
$$

where $q$ is the convection heat flux $\left(W / m^{2}\right), h$ the heat convection coefficient $\left(\mathrm{W} / \mathrm{m}^{2} /{ }^{\circ} \mathrm{C}\right)[10], T_{H e}$ the 
temperature of $\mathrm{He}$ atmosphere (known to be equal to $\left.30^{\circ} \mathrm{C}\right)$.

The specimen is initially heated to a central temperature at mid-thickness of $400^{\circ} \mathrm{C}$ using a low voltage, high current electrical power supply. This process is modeled by introducing only the Joule effect term, which is calibrated by adjusting the value of the volumetric source $s$ so that the temperature at the central impacted zone stabilizes at $400^{\circ} \mathrm{C}$ after several minutes of transient phase. It is found that $s=3.36 \times$ $10^{7} \mathrm{~W} \mathrm{~m}^{-3}$, which is consistent with the experimental settings with $3 \mathrm{~kW}$ electrical power. A temperature of $100^{\circ} \mathrm{C}$ is prescribed at the end of the simulated volume, which corresponds to the level measured at midthickness under the copper clamps during the test.

The resulting temperature field is introduced as the initial conditions. Then the cyclic thermal loading on the central zone of the surface is modeled by a super Gaussian profile [11]

$$
I(y, z, p)=\alpha I_{0} \frac{p 4^{\frac{1}{p}}}{2 \pi \Gamma\left(\frac{2}{p}\right)} \exp \left(-2 r(y, z)^{p}\right)
$$

where $\alpha$ is the surface absorptivity, $I_{0}$ the chosen peak power of laser pulse known to be equal to $310 \mathrm{~W}, \Gamma$ the Gamma function, $p$ the shape parameter and $r$ the reduced cross-sectional distance

$$
r^{2}(y, z)=\left(\frac{y}{R_{y}}\right)^{2}+\left(\frac{z}{R_{z}}\right)^{2}
$$

The parameters of the spatial power density distribution of the laser beam are determined by comparing experimental (as shown in Fig. 3) and numerical temperature fields from a thermal FE model updating procedure [12].

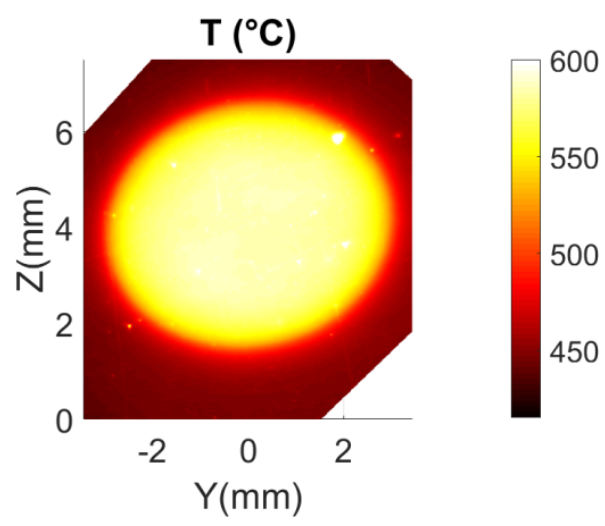

Fig. 3. Surface temperature field measured by IR camera at the end of a laser pulse

The values of identified parameters are summarized in Tab. 1.

Table 1. Identified parameters of spatial power density distribution of laser pulse

\begin{tabular}{|c|c|c|c|}
\hline$\alpha$ & $p$ & $R_{y}(\mathrm{~mm})$ & $R_{z}(\mathrm{~mm})$ \\
\hline 0.51 & 12 & 3.08 & 2.96 \\
\hline
\end{tabular}

Based on the information described above, the temperature field at the end of a laser pulse calculated by the $3 \mathrm{D}$ transient model is shown in Fig. 4. The distribution of temperature resulting from the heating up phase is illustrated outside the zone impacted by the laser pulse. It shows that the temperature gradient is particularly steep along the thickness direction (labelled as $X$ for the FE model), and lower along the other two directions in the plane of specimen surface (labelled as $Z$ along the longitudinal direction, and $Y$ along the transverse direction of the FE model), see Fig. 5.

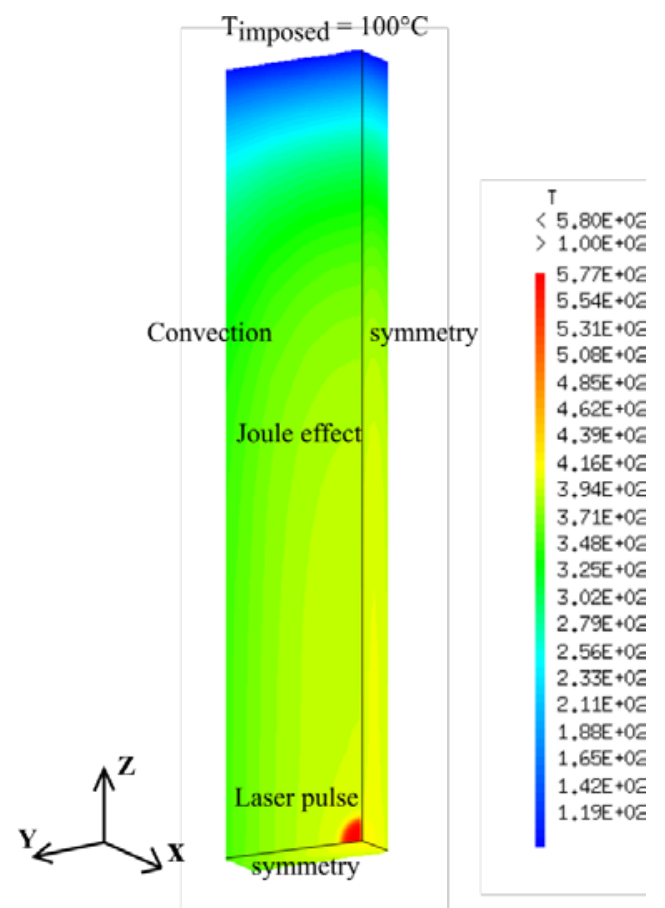

Fig. 4. 3D Temperature field at the end of a laser pulse with a prescribed temperature amplitude of $170^{\circ} \mathrm{C}$ in the central zone

This feature is directly linked to the relatively short laser pulse duration $(50 \mathrm{~ms})$, the surface temperature change has occurred long before the shock effects have penetrated significantly through the thickness. A disk of almost $2 \mathrm{~mm}$ in radius can be considered as being shocked with the same temperature variation and from which fatigue cracks initiate and propagate, which is confirmed from the optical microscopy observations reported in Section 4.

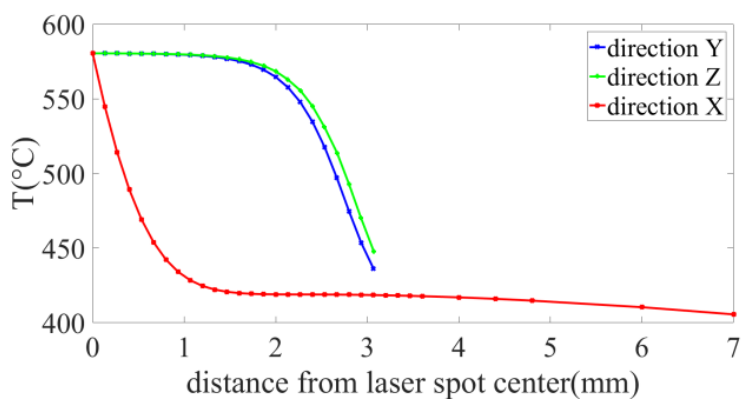

Fig. 5. Temperature gradient along three directions in the central beam impacted zone, at the end of a laser pulse 


\subsection{Mechanical analyses}

The change of temperature induces significant thermal strain variations in the central part of the impacted zone, while the bulk remains in the initial state; such local expansions are then hindered by the bulk that is under tension during this laser pulse activation stage.

Thus a thermomechanical analysis is run by applying the stabilized cyclic thermal loading with the selected boundary conditions. First, there is no externally applied stress onto the specimen; and with symmetry considerations, no displacement on axial and transverse surfaces is expected.

With the assumption that the generated strain amplitudes are moderate, the total strain tensor is decomposed as follows

$$
\underline{\varepsilon}^{\text {total }}=\underline{\varepsilon}^{\text {meca }}+\underline{\varepsilon}^{\text {ther }}
$$

where the thermal strain tensor is defined as

$$
\underline{\underline{\varepsilon}}^{\text {ther }}=\alpha_{T} \cdot\left(T-T_{i n i}\right) \underline{\underline{I}}
$$

and $\alpha_{T}$ is the thermal expansion coefficient, $\left(T-T_{i n i}\right)$ the variation of temperature, and $\underline{I}$ the identity tensor. The change of $\alpha_{T}$ as a function of temperature is extracted from the French design code RCC-MRx [9].

Within the small strain framework, the mechanical strain tensor is decomposed into elastic and plastic contributions

$$
\underline{\underline{\varepsilon}}^{\text {meca }}=\underline{\underline{\varepsilon}}^{\text {elas }}+\underline{\underline{\varepsilon}}^{\text {plas }}
$$

The elastic domain is defined by

$$
f=J_{2}(\underline{\underline{\sigma}}-\underline{\underline{X}})-R(T) \leq 0
$$

in which $J_{2}$ is the second invariant of the deviatoric stress tensor, $R$ the temperature dependent initial yield stress, and $\underline{X}$ the back-stress tensor.

As proposed in Ref. [3], a stabilized stress-strain behavior can be obtained after a short transient period; the cyclic stress change is described using a series of hardening laws. The thermomechanical behavior of the material is then described by a nonlinear kinematic hardening law [13]

$$
\underline{\underline{X}}=\sum_{i=1}^{N=2} \underline{\underline{X_{i}}}
$$

where each kinematic variable $X_{i}$ follows the growth law

$$
\underline{\underline{X_{l}}}=C_{i}\left(\frac{2}{3} A_{i} \underline{\underline{\dot{\varepsilon}^{p}}}-\underline{\underline{X}} \underline{\underline{\underline{p}}}\right)
$$

The thermomechanical parameters $R, C_{i}$ and $A_{i}$ are calibrated on push-pull cyclic tests at $400^{\circ} \mathrm{C}$ and $500^{\circ} \mathrm{C}$ [12]. A consistent set of parameters is then obtained in which only $R$ is a (linear) function of the temperature as

$$
R(T)=73.49-0.0735 T
$$
2.

The values of identified parameters are listed in Tab.

Table 2. Identified parameters for non-linear kinematic hardening laws

\begin{tabular}{|c|c|c|c|}
\hline$A_{1}(M P a)$ & $C_{1}$ & $A_{2}(M P a)$ & $C_{2}$ \\
\hline 105.8 & 684.5 & 235.6 & 15.3 \\
\hline
\end{tabular}

Mechanical simulations present nearly perfect equibiaxial stress distributions on the surface center as shown in Fig. 6.

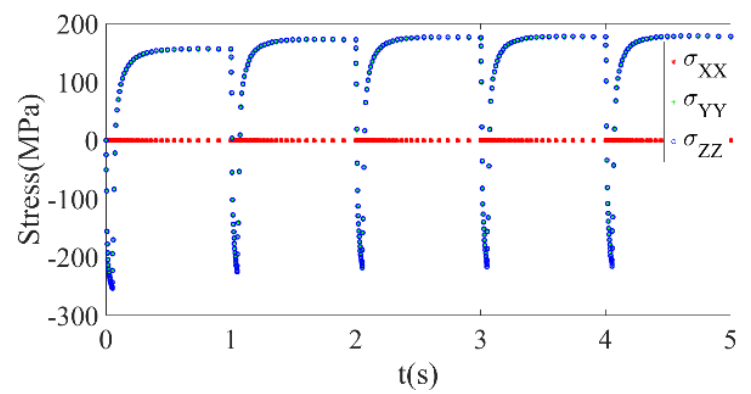

Fig. 6. Simulated stress history in the central zone of the specimen surface impacted by the laser beam

The histories of eigen strains (simulated and experimentally measured) are compared in Fig. 7. The levels of total strains measured by multiview correlation are well reproduced by the simulations along the two eigen directions in the plane of specimen surface.

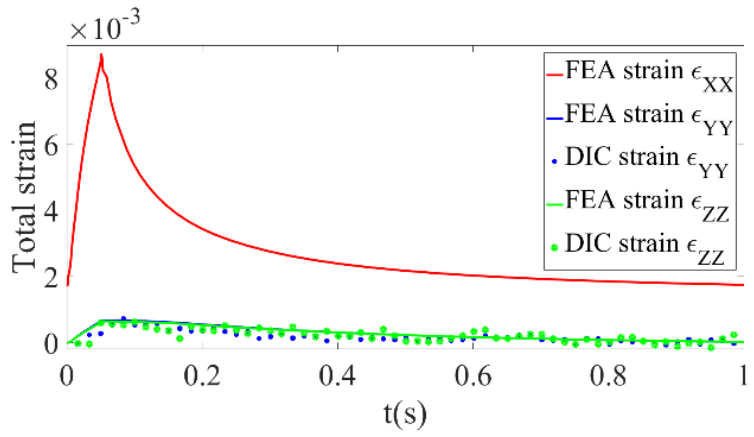

Fig. 7. Strain history in the central zone of the surface impacted by the laser beam. Solid lines stand for 3D simulation results, and solid symbols for experimental measurements

Moreover, the changes of out of plane displacement induced by the largest strain variation $\epsilon_{X X}$ during cyclic loading are consistent with the experimental measurements (Fig. 8).

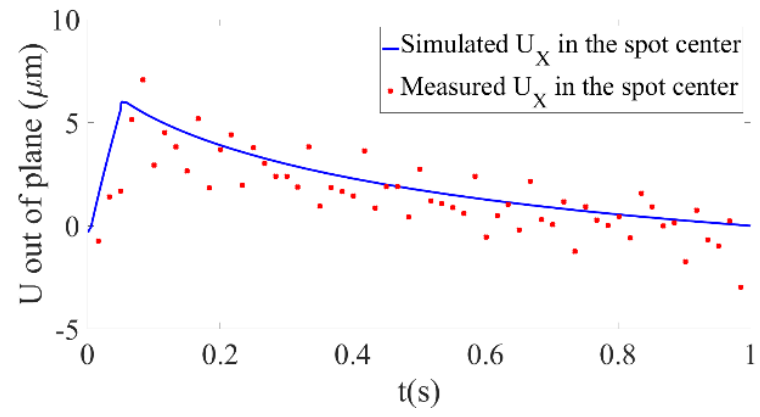

Fig. 8. Out of plane displacement in the central zone of the surface impacted by the laser spot. The solid line stands for 3D simulation results, and solid symbols for experimental measurements 


\section{Fatigue analysis and comparison with results from uniaxial isothermal fatigue tests}

Since the out-of-plane strain variation is not experimentally accessible during thermal fatigue tests, the equivalent Tresca strain variation extracted from mechanical simulations is needed in order to interpret the thermal fatigue experiments and compare them with isothermal mechanical fatigue tests.

The Tresca equivalent total strain range is calculated by adding the contributions of the Tresca equivalent elastic and plastic strain ranges where the plastic strain range can be obtained with a methodology based on the correction of the Poisson effect in the elastic analysis of low cycle fatigue

$$
\Delta \varepsilon_{\text {Tresca }}^{\text {tot }}=\Delta \varepsilon_{\text {Tresca }}^{\text {elastic }}+\Delta \varepsilon_{\text {Tresca }}^{\text {plastic }}
$$

with

$$
\Delta \varepsilon_{\text {Tresca }}^{e, p}=\frac{1}{1+v} \max \left(\left|\Delta \varepsilon_{1}^{e, p}-\Delta \varepsilon_{2}^{e, p}\right|,\left|\Delta \varepsilon_{1}^{e, p}-\Delta \varepsilon_{3}^{e, p}\right|,\left|\Delta \varepsilon_{2}^{e, p}-\Delta \varepsilon_{3}^{e, p}\right|\right)
$$

where $v$ is the Poisson's ratio equal to 0.3 and 0.5 for elastic and plastic calculations, respectively. For this set of simulation, the Tresca equivalent total strain range is $0.45 \%$ in the central laser beam zone for a temperature variation of $170^{\circ} \mathrm{C}$.

The number of cycles to initiate cracks in the fatigue test is deduced from singularities in the gray level residual maps of the correlation technique. The main fatigue crack initiates after 80,000 cycles (Fig 9). This sample has been observed periodically by optical microscopy during the thermal fatigue test in order to investigate the formation and growth of fatigue cracks at a scale hardly detectable by correlation techniques. After only 6,000 cycles, very intense slip bands are observed on the surface within the shocked region, and several microcracks with length of $50 \mu \mathrm{m}$ begin to initiate in the intersection of slip bands with grain boundaries.

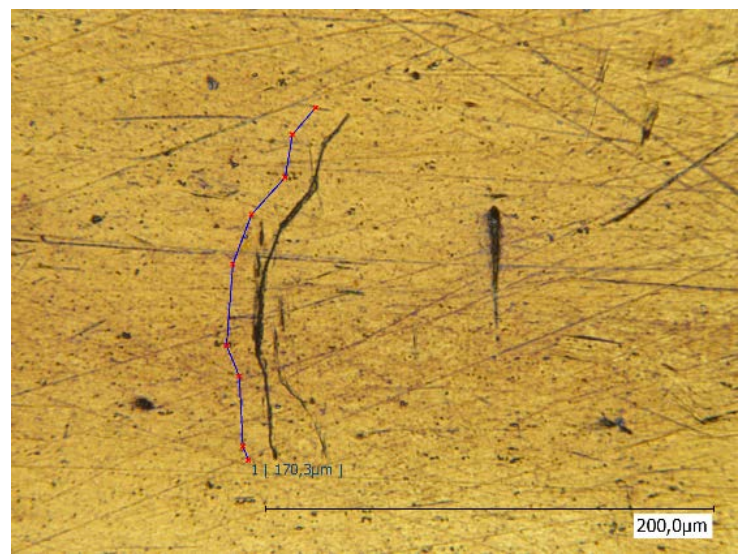

Fig. 9. Main fatigue crack with a length of $170 \mu \mathrm{m}$ after 80,000 cycles for a thermal loading $\Delta \mathrm{T}=170^{\circ} \mathrm{C}$ observed by optical microscopy
The main fatigue crack is located just in the central zone of the surface impacted by the laser beam and initiated during the first 26,000 cycles. Then it propagated as shown in Fig. 10 until a $170 \mu \mathrm{m}$ crack length is clearly observed after 80,000 cycles. A second crack with another orientation (perpendicular to the first one) is observed after 42,000 cycles with an initial length of $40 \mu \mathrm{m}$, and then it has grown up to $90 \mu \mathrm{m}$ after 80,000 cycles. These observations confirm the biaxial state induced by thermal loading and the initiation of cracks on permanent slip bands induced by the plastic strains on the sample surface.

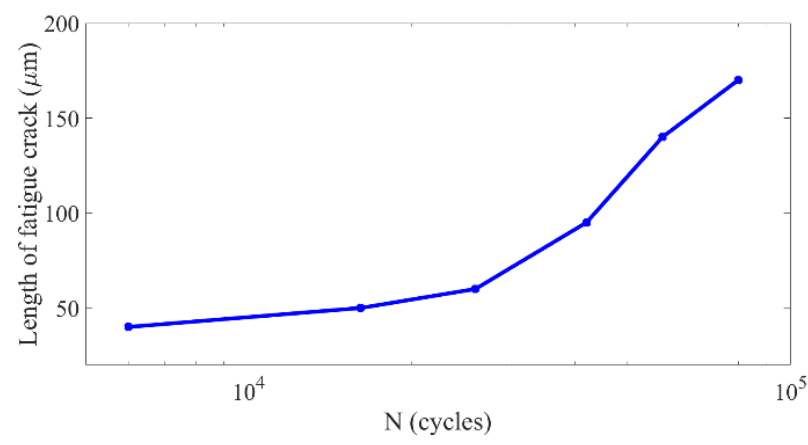

Fig. 10. Propagation of the main fatigue crack with a thermal loading $\Delta \mathrm{T}=170^{\circ} \mathrm{C}$ observed by optical microscopy

In Fig. 11, the thermal fatigue results are compared with uniaxial isothermal fatigue data obtained at $400^{\circ} \mathrm{C}$ and $500^{\circ} \mathrm{C}$ for the same material. The ordinate of the graph is the Tresca strain variation, while the abscissa is the number of cycles to "initiate" $200 \mu \mathrm{m}$ cracks in thermal fatigue tests or the number of cycles to get a $25 \%$ decrease of the maximum stress in push-pull fatigue tests performed on $8 \mathrm{~mm}$ cylindrical specimen.

With the FLASH data it is concluded that under the same equivalent strain ranges, that is to say under the same mechanical loading, the environmental effect has a minor influence on the fatigue life. This observation validates the explanations $[14,15]$ that the environmental effect is negligible compared to strain aging and dislocation motion at high temperatures (i.e., in ranges from $300^{\circ} \mathrm{C}$ to $800^{\circ} \mathrm{C}$ ) for $316 \mathrm{~L}$ austenitic stainless steel, especially for the frequency of pulsed laser ( $1 \mathrm{~Hz}$ in FLASH tests) that leads to environmental interactions barely able to develop. Besides, FLASH results show higher number of cycles to initiation compared with uniaxial isothermal fatigue tests under the same strain range $(0.5 \%)$. This difference may result from the mean bi-compressive stress states induced by hot thermal shocks, which is favorable for the fatigue resistance [16]. Yet it should be noted that this comparison is incomplete since push-pull fatigue test data under higher strain ranges are not available. Overall, the present thermal fatigue data are in good agreement with classical fatigue test results and are located well above the design curve of the RCC-MRx design code. 


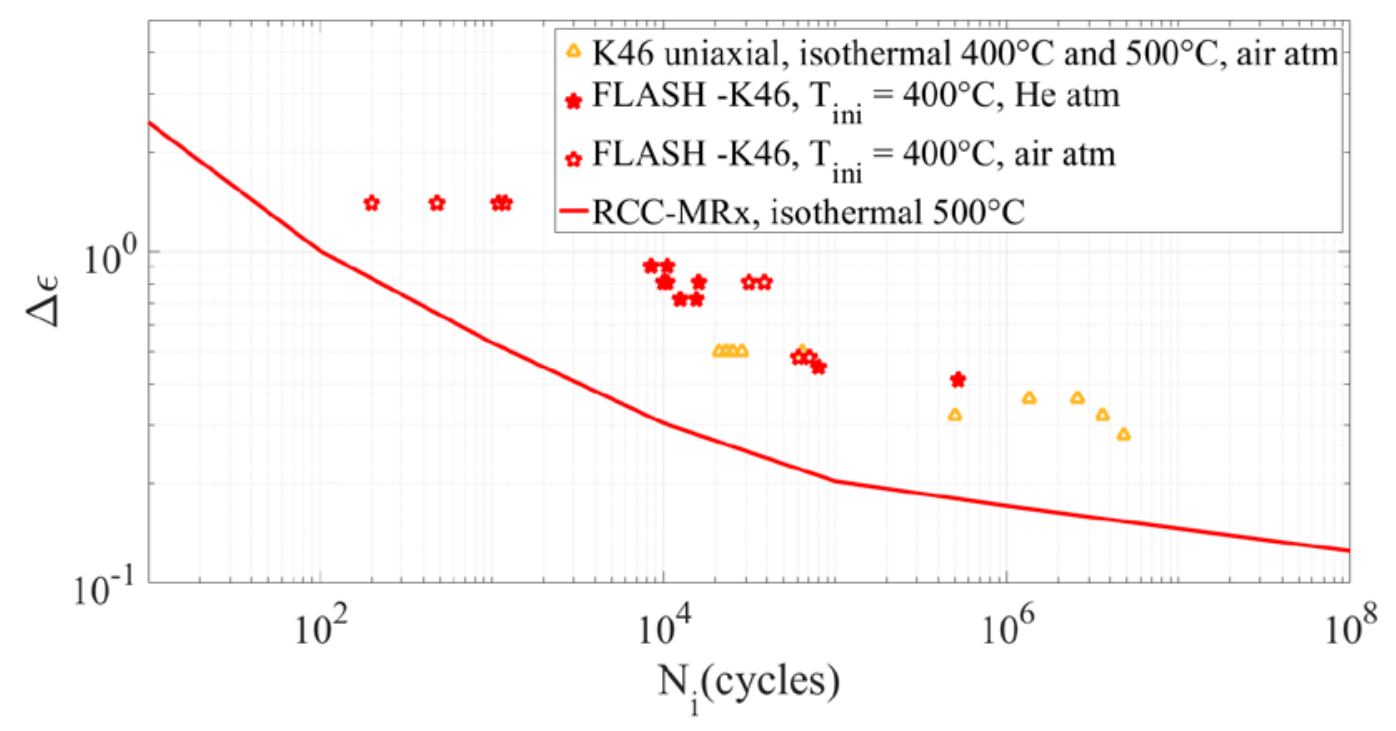

Fig. 11. Equivalent Tresca strain range as a function of number of cycles required to initiate $200 \mu \mathrm{m}$ cracks with FLASH (red symbols). Comparaison with number of cycles for isothermal fatigue tests (orange symbols) and with the fatigue design curve of RCC-MRx design code (solid line)

\section{Conclusion}

The FLASH facility allows thermal fatigue tests to be performed on specimen made of $316 \mathrm{~L}(\mathrm{~N})$ austenitic stainless steel with highly stable thermal amplitudes ranging from $150^{\circ} \mathrm{C}$ to $200^{\circ} \mathrm{C}$ prescribed by pulsed laser shocks. Full-field temperature and displacement measurements on the surface of the specimen are carried out by using infrared and visible light cameras. The equivalent Tresca strain range obtained from numerical simulations combined with crack initiation detection via correlation enable the fatigue strength of the studied material to be estimated.

The comparison with the French design code RCCMRx shows a good conservative agreement. The next tests will be conducted with different thermal loading histories to build up an extensive and well-documented database. Furthermore, tests with helium pulses onto hot plates or with static 4-point bending should be performed to investigate the mean bi-tensile stress effect on the fatigue response.

\section{References}

1. P. Le Coz, J. Sauvage, J. P. Serpantié, Proceedings of ICAPP 2851. (France, 2011)

2. N. Robert, J. Economou, F. Cornuel, O. Volte, J.-M. Stephan, International symposium on the contribution of materials investigations to improve the safety and performance of LWRs (Fontevraud, France, 2006)
3. A. Fissolo, S. Amiable, O. Ancelet, F. Mermaz, J. M. Stelmaszyk, A. Constantinescu, C. Robertson, L. Vincent, F. Bouchet, Int J Fatigue, 31(3), 587-600. (2009)

4. A. Fissolo, S. Amiable, O. Ancelet, F. Mermaz, J. M. Stelmaszyk, A. Constantinescu, C. Robertson, L. Vincent, F. Bouchet, Int J Fatigue, 31(7), 1196-1210. (2009)

5. L. Vincent, M. Poncelet, S. Roux, F. Hild, D. Farcage, Procedia Eng, 66, 669-675. (2013)

6. A. Charbal, L. Vincent, F. Hild, M. Poncelet, J. E. Dufour, S. Roux, D. Farcage, QIRT J, 13(1), 1-18. (2016)

7. A. Charbal, J. E. Dufour, F. Hild, M. Poncelet, L. Vincent, S. Roux, Exp. Mech, 56(5), 845-860. (2016)

8. Y. Wang, A. Charbal, J. E. Dufour, F. Hild, S. Roux, L. Vincent, Photomechanics, (France, 2018)

9. AFCEN, RCC-MRx (2012).

10. F. W. Giacobbe, Appl. Therm. Eng, 25(2), 205-225. (2005)

11. D. L. Shealy, J. A. Hoffnagle, Appl. Opt, 45(21), 51185131. (2006)

12. A. Charbal, $P h D$ thesis, (University of Paris-Saclay, 2017)

13. J.-L. Chaboche, Int J Plasticity, 24(10), 1642-1693. (2008)

14. R. Alain, P. Violan, J. Mendez, Mater. Sci. Eng. A, 229(12), 87-94. (1997)

15. K. Sadananda, P. Shahinian, Metall. Mater. Trans. A, 11(2), 267-276. (1980)

16. L. Vincent, $H D R$, (Ecole Normale Supérieure de Cachan, 2012)

\footnotetext{
Corresponding author: ludovic.vincent@ece.fr
} 